\title{
Spectrum- and RGB-D-Based Image Fusion for the Prediction of Nitrogen Accumulation in Wheat
}

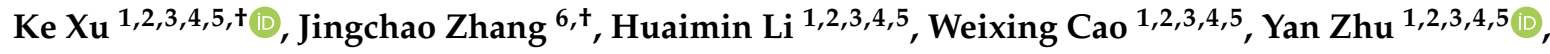 \\ Xiaoping Jiang $1,2,3,4,5$ and Jun $\mathrm{Ni}^{1,2,3,4,5, *}$ \\ 1 College of Agriculture, Nanjing Agricultural University, Nanjing 210095, China; \\ 2017101174@njau.edu.cn (K.X.); 2019101174@njau.edu.cn (H.L.); caow@njau.edu.cn (W.C.); \\ yanzhu@njau.edu.cn (Y.Z.); xpjiang@njau.edu.cn (X.J.) \\ 2 National Engineering and Technology Center for Information Agriculture, Nanjing 210095, China \\ 3 Engineering Research Center of Smart Agriculture, Ministry of Education, Nanjing 210095, China \\ 4 Jiangsu Key Laboratory for Information Agriculture, Nanjing 210095, China \\ 5 Jiangsu Collaborative Innovation Center for the Technology and Application of Internet of Things, \\ Nanjing 210095, China \\ 6 Nanjing Institute of Agricultural Mechanization, Ministry of Agriculture and Rural Affairs, \\ Nanjing 210014, China; zhangjingchao@caas.cn \\ * Correspondence: nijun@njau.edu.cn; Tel.: +86-25-8439-6593 \\ + These authors contributed equally to this work.
}

Received: 16 November 2020; Accepted: 8 December 2020; Published: 10 December 2020

\begin{abstract}
The accurate estimation of nitrogen accumulation is of great significance to nitrogen fertilizer management in wheat production. To overcome the shortcomings of spectral technology, which ignores the anisotropy of canopy structure when predicting the nitrogen accumulation in wheat, resulting in low accuracy and unstable prediction results, we propose a method for predicting wheat nitrogen accumulation based on the fusion of spectral and canopy structure features. After depth images are repaired using a hole-filling algorithm, RGB images and depth images are fused through IHS transformation, and textural features of the fused images are then extracted in order to express the three-dimensional structural information of the canopy. The fused images contain depth information of the canopy, which breaks through the limitation of extracting canopy structure features from a two-dimensional image. By comparing the experimental results of multiple regression analyses and BP neural networks, we found that the characteristics of the canopy structure effectively compensated for the model prediction of nitrogen accumulation based only on spectral characteristics. Our prediction model displayed better accuracy and stability, with prediction accuracy values $\left(R^{2}\right)$ based on BP neural network for the leaf layer nitrogen accumulation (LNA) and shoot nitrogen accumulation (SNA) during a full growth period of 0.74 and 0.73 , respectively, and corresponding relative root mean square errors (RRMSEs) of $40.13 \%$ and $35.73 \%$.
\end{abstract}

Keywords: RGB-D image; fused image; spectrum; wheat nitrogen accumulation

\section{Introduction}

Nitrogen is a key element required for the growth and development of wheat, and thus exerts an important influence on its yield and quality. At present, during wheat production, in order to pursue high crop yields, problems exist such as excessive nitrogen fertilizer application and unscientific fertilization methods, which not only cause environmental pollution, but also reduce profits [1-3]. Therefore, the precise management of wheat fertilization and the realization of scientific nitrogen application not only have the production significance of saving nitrogen and increasing efficiency, but also the ecological significance of reducing farmland pollution. 
A quick and effective grasp of the status of nitrogen nutrition indicators during the key growth period of wheat is a prerequisite for achieving accurate fertilizer management. With the continuous development of spectral analysis and image sensing, the timeliness of the non-destructive testing of nitrogen nutrition indices is steadily improving, and the testing method is becoming increasingly convenient [4-7]. The premise of spectral analysis technology is primarily to simplify and idealize the crop canopy as an isotropic and uniform Lambertian surface. In previous research, there have been many investigations on the real-time estimation of nitrogen nutrition indices using multi-spectral and hyper-spectral remote sensing equipment [5,8-11], reaching conclusions including that the sensitive bands of crop nitrogen nutrition status are mainly concentrated in the visible and near-infrared bands, with the "red edge" area being the most prominent [12,13]. Existing research on the interaction mechanism of the vegetation canopy and radiation, however, indicates that the assumption that the vegetation canopy should be regarded as a Lambertian surface is quite different from the actual situation. When the influence of the anisotropic characteristics of canopy structure on the spectrum is ignored, the detection results are not stable enough, and the accuracy is relatively poor $[14,15]$. Therefore, it is necessary to combine spectral characteristics and canopy structure characteristics in order to detect the nitrogen nutrition status of crops, because the combination of the two characteristics can have a more positive impact on the predicted results. At present, the extraction of canopy structure features mostly relies on digital image processing technology, and the differences in color and morphological characteristics of the crop canopy are expressed by analyzing image features [16-18]. Since only visible light band information is used, the R, G, and B values of the image are easily affected by lighting conditions at the time the image is captured, and, since it is difficult for a two-dimensional digital image to reflect the three-dimensional morphological information of the crop canopy, the accuracy of constructing a nitrogen nutrition index estimation model based on two-dimensional digital images is not ideal [19].

In recent years, depth information has gradually been applied to the three-dimensional reconstruction of plants and the monitoring of crop growth, which has the advantage of quickly reconstructing the three-dimensional structure of crops [20]. Compared with RGB and grayscale images, depth images contain more accurate geometric information of the features. Consumer-grade RGB-D cameras provide an inexpensive solution for the rapid acquisition of 3D crop information. Existing studies have shown that RGB-D cameras provide a convenient way to quickly obtain crop canopy structure information in a field environment [21,22]. The applications of RGB-D cameras include extracting crop canopy structure parameters through three-bit reconstruction $[23,24]$, and using height differences to remove weed information [25].

Because spectral characteristics are affected by canopy structure during the prediction of nitrogen accumulation in wheat, the model prediction accuracy is not high. When extracting canopy structure parameters, RGB images cannot reflect the three-dimensional structural characteristics of the canopy, while depth images can better reflect the three-dimensional structural information of crops [23]. In order to consider both RGB images and depth images in the construction of canopy structure features, we propose a pixel-level fusion algorithm of RGB images and depth images based on the correlation between the corresponding pixels of the two image types. By constructing characteristic parameters that combine the color and structural information of the wheat canopy, we compensate for the previous model limitations when predicting the leaf layer nitrogen accumulation (LNA) and shoot nitrogen accumulation (SNA) of wheat with a single spectral feature, and establish a predictive model based on multi-dimensional features.

\section{Materials and Methods}

\subsection{Wheat Experiment Design}

From December 2017 to April 2018, a wheat experiment (Figure 1) was conducted at the Demonstration Base of the National Engineering and Technology Center for Information Agriculture 
in Rugao City $\left(32^{\circ} 16^{\prime} \mathrm{N}, 120^{\circ} 46^{\prime} \mathrm{E}\right)$, Nantong City, Jiangsu Province, China. The wheat variety selected for the experiment was Shengxuan 6 , and the experiment was divided into three nitrogen treatments, namely N0 (0 kg N/ha), N1 (180 kg N/ha), and N2 (360 kg N/ha). The planting densities had row spacings of $20 \mathrm{~cm}, 35 \mathrm{~cm}$, and $50 \mathrm{~cm}$. Three replicates were set for each group of experiments, for a total of 27 plots, with the area of each plot being $5 \times 6 \mathrm{~m}^{2}$.

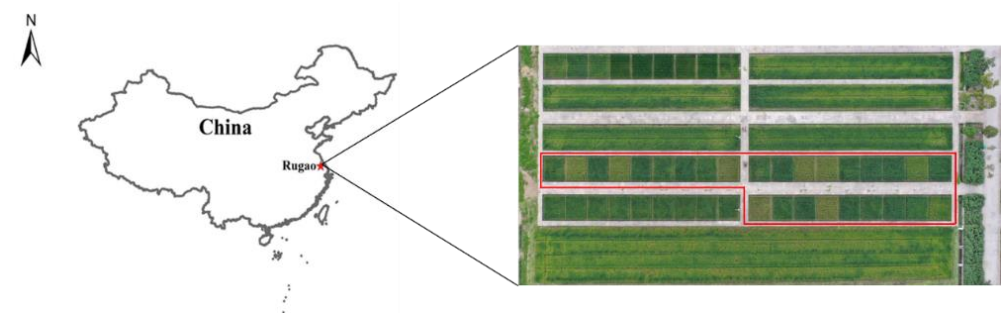

Figure 1. Wheat test scene, in which the red box is the wheat test plot.

\subsection{Obtaining Wheat Agronomic Parameters}

Based on the key fertilization periods of wheat, samples were taken at the jointing, booting, and heading stages. Due to the relatively long duration of the jointing stage, two samples were taken during this stage. From each plot, we randomly selected 15 wheat plants for sample analysis, and then separated them indoors according to their organs (stems, leaves, and ears). After the samples were fixed at $105^{\circ} \mathrm{C}$ for $30 \mathrm{~min}$, they were baked to a constant weight at $80^{\circ} \mathrm{C}$ and then weighed. The individual organ weights, i.e., the leaf dry weight $(L D W)$, stem dry weight $\left(S_{\text {tem }} D W\right)$, and spike dry weight $\left(S_{\text {pike }} D W\right)$, were measured. After each sample was crushed, the Kjeldahl method was used to determine the leaf nitrogen content $(L N C)$, stem nitrogen content $\left(S_{t e m} N C\right)$, and spike nitrogen content $\left(S_{\text {pike }} N C\right)$. We then calculated the leaf layer nitrogen accumulation $(L N A)$ and shoot nitrogen accumulation (SNA) according to the following equations.

$$
\begin{gathered}
\operatorname{LNA}\left(\mathrm{g} / \mathrm{m}^{2}\right)=\operatorname{LNC}(\%) \times \operatorname{LDW}\left(\mathrm{g} / \mathrm{m}^{2}\right) \\
S N A\left(\mathrm{~g} / \mathrm{m}^{2}\right)=\operatorname{LNA}\left(\mathrm{g} / \mathrm{m}^{2}\right)+S_{\text {tem }} N C(\%) \times S_{\text {tem }} D W\left(\mathrm{~g} / \mathrm{m}^{2}\right)+S_{\text {pike }} N C(\%) \times S_{\text {pike }} D W\left(\mathrm{~g} / \mathrm{m}^{2}\right)
\end{gathered}
$$

\subsection{Wheat Spectrum and Image Data Acquisition}

The ASD FieldSpec HandHeld 2 was used to obtain the spectral data of the crop. The measurement position was $1 \mathrm{~m}$ from the top of the canopy. Each plot was measured three times at different locations, with the average value representing the spectral information of the plot. We used the Intel ${ }^{\circledR}$ RealSense $^{\mathrm{TM}}$ D415 Depth Camera to acquire wheat field images. As shown in Figure 2b, the camera has two infrared stereo cameras, one infrared emitter, and one color sensor. The infrared stereo camera was used to generate depth images, and the color sensor was used to generate RGB images, both with a resolution of $1280 \times 720$ pixels. The camera was positioned $70 \mathrm{~cm}$ from the crop canopy, and we selected three different locations in each plot for image acquisition. The actual image acquisition device in the field is shown in Figure 2.

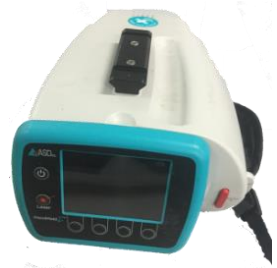

(a)

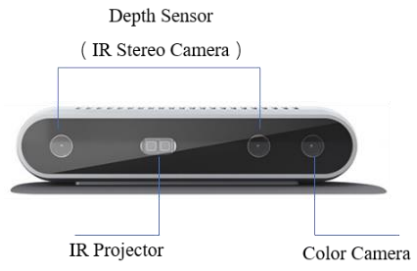

(b)

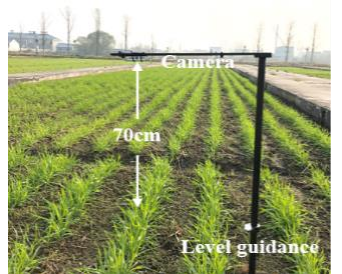

(c)

Figure 2. (a) ASD FieldSpec HandHeld 2; (b) Intel ${ }^{\circledR}$ RealSense ${ }^{\mathrm{TM}}$ D415 Depth Camera; (c) image acquisition device. 


\subsection{RGB-D Image Preprocessing}

\subsubsection{Depth Image Restoration Method}

Due to lighting conditions, infrared reflection characteristics of the surface material of the measured object, and area occlusion, depth images have the problem of missing data (appearing as holes), resulting in decreased accuracy of the target object information, which affects the subsequent feature extraction. As shown in Figure 3, we divide the occurrence of holes into two categories for discussion: local holes and large-area holes. Local holes appear within the leaf, and are mainly affected by the surrounding environmental conditions, such as light and vibration. The area of this type of hole is usually small, and the depth information is similar to the surrounding depth information. Large-area holes appear at the junctions of different areas. The reason for their occurrence is related to the application range of the sensor. They mainly appear at the transition of depth information on the edge of the leaf. Based on the analysis of different types of holes, we designed a hole-filling algorithm (HF), consisting of the following steps. First, perform column traversal on the depth information of a crop row image, find the positions of all the hole points (line), and calculate the difference $\mathrm{H}$ of the non-zero depth information before and after the point (line). By comparing $\mathrm{H}$ with an artificially set threshold $\mathrm{T}$, the types of holes are distinguished. When $\mathrm{H}<\mathrm{T}$, it is a local hole, and the mean value of the non-zero depth information before and after the hole point (line) is interpolated. When $\mathrm{H} \geq$ $\mathrm{T}$, it is a large-area hole, and the non-zero depth information before and after the hole point (line) is separately fitted and interpolated. Since there were horizontal crop rows in the center of the images we examined, a curve was more suitable for fitting. We used a quadratic polynomial for fitting. HF is expressed as Equation (3), where $H b$ and $H f$ represent the non-zero depth information before and after the hole point (line), respectively; $x$ represents the column coordinates corresponding to the hole point; and $d(x)$ is the depth information to be filled.

$$
d(x)=\left\{\begin{array}{l}
\frac{H b+H f}{2} H<T \\
a x^{2}+b x+c H \geq T
\end{array} .\right.
$$

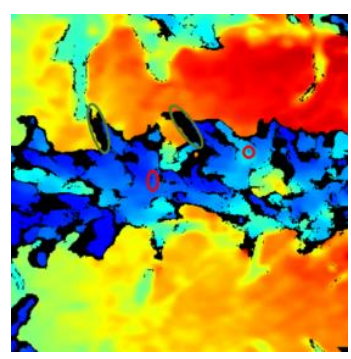

(a)

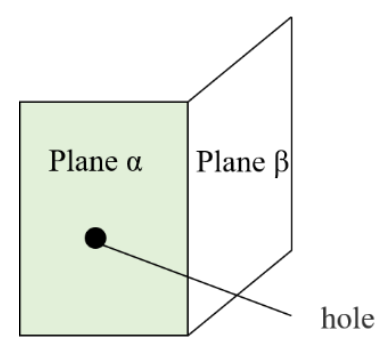

(b)

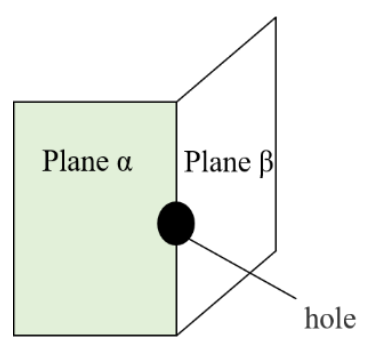

(c)

Figure 3. (a) Depth image of the wheat field, in which the red circles represent local holes and green circles represent large-area holes; (b) local hole within the area; (c) large-area hole at the junction of different areas.

\subsubsection{Wheat Canopy Segmentation Method Based on RGB-D Images}

There are non-crop canopy features such as soil, straw, and weeds in canopy images of wheat during its early and mid-term growth. In order to eliminate the interference of non-canopy information in the images during feature extraction, the canopy portion of the images needs to be segmented and extracted. Common vegetation indices such as ExG and ExGR are greatly affected by environmental light when performing canopy segmentation and cannot remove green information such as weeds. When the light is strong, the color of the reflective leaf is closer to the color of the soil background, and is thus easily removed by mistake, resulting in poor segmentation. In order to avoid the influence of ambient light on the segmentation results, we propose segmenting the soil background in hue, 
saturation, lightness (HSI), and space. The value range of the $\mathrm{H}$ channel in the HSI color model is $[0,2 \pi]$, and the $H$ values corresponding to yellow, green, and cyan are $\frac{\pi}{3}, \frac{2 \pi}{3}$, and $\pi$, respectively. Due to different nitrogen treatments of wheat, the colors of the leaves varied, so the $H$ channel value in the interval $\left(0, \frac{4 \pi}{3}\right)$ was retained as crop row information. In addition, considering the influence of field weed information on the extraction of crop row characteristics-since the wheat closes the rows after the jointing stage-weeds no longer affect the subsequent extraction of wheat canopy characteristics, and were only removed during the jointing stage via height information obtained from the depth images.

\subsubsection{RGB-D Image Fusion Method for the Wheat Canopy}

When most studies use RGB images to obtain canopy structure information, they extract coverage and other information through color differences, which cannot express the characteristics of wheat canopy structure in a three-dimensional space [26]. When using depth information for crop growth parameter inversion, researchers generally extract canopy height information to predict above-ground biomass and other agronomic parameters, and no further methods for extracting canopy structure features based on three-dimensional space have been proposed [27]. In order to simultaneously fuse RGB information and depth information to express the characteristics of canopy structure, we propose a pixel-level RGB-D image fusion method, which uses the connection between the corresponding pixels of RGB images and depth images to complement the two kinds of information in the modeling process.

Since the imaging origins of RGB images and depth images are inconsistent, depth information and RGB image information cannot correspond one-to-one. For the subsequent feature extraction, the first step is to align two images. The basic idea of image alignment is the conversion of different coordinate systems. By converting the coordinate system of a depth image to the world coordinate system and then to the coordinate system of an RGB image, the alignment of the two images is realized.

The basic idea of the RGB-D image fusion method is to use the physical relationship between intensity and phase. In the natural environment, color information in an RGB image indicates the reflection intensity of the object, and the intensity information is affected by different lighting conditions. The principle of acquiring depth information is that the sensor collects different phase information for different depth areas of the subject, and then converts these structural variations into depth information through the arithmetic unit. Therefore, the inverse square law can be used to describe the relationship between intensity and phase [28]:

$$
d \approx I_{R} / \phi^{2},
$$

where $I_{R}$ represents the intensity information of each point in the RGB image, corresponding to the grayscale value after the RGB image is converted to a grayscale image, and $d$ represents the depth information corresponding to $I_{R}$. From the inverse square law, the phase information $\phi$ of each pixel in the image can be calculated. When performing image fusion, we use the idea of IHS transform to replace the I component in the HSI model with the phase information $(\phi)$ and depth information $(d)$ for representation, thus obtaining a fused image based on HSP (where P is phase information) and HSD (where D is depth information) models. Since the value range of the I channel is $[0,1]$, in order to simplify the calculation, the depth information is normalized. The normalization formula is as follows:

$$
\bar{d}=\left(d-d_{\min }\right) /\left(d_{\max }-d_{\min }\right),
$$

where $d$ is the actual depth information, $d_{\max }$ and $d_{\min }$ are the maximum and minimum depth information, respectively, and $\bar{d}$ represents the normalized results. 


\subsection{Wheat Canopy Feature Extraction}

\subsubsection{Spectral Feature Extraction}

Previous studies have shown that the spectral reflectance of crops at $730 \mathrm{~nm}$ and $815 \mathrm{~nm}$ performs well in assessing leaf area index, pigment status, and nitrogen nutrition status [29,30]. Therefore, we selected the above two bands to construct the normalized difference red edge (NDRE) index, and the ratio vegetation index (RVI). The calculation formulas of vegetation indices are as follows:

$$
\begin{gathered}
N D R E=(R 815-R 730) /(R 815+R 730), \\
R V I=R 815 / R 730,
\end{gathered}
$$

where $R 730$ and $R 815$ represent the reflectance of wheat in the 730 and $815 \mathrm{~nm}$ bands, respectively.

\subsubsection{Feature Extraction of Fused Images}

In order to represent the information of the wheat canopy structure more accurately, a single row of crops was intercepted for feature extraction, and the crop row image block size intercepted in all periods was $150 \times 500$ pixels. After image fusion, the color co-occurrence matrix was used to extract texture features. The color co-occurrence matrix usually contains a large amount of information. In order to reduce the dimensionality of the feature space as much as possible to decrease the amount of calculation, while still retaining the description of the image texture information, we selected five feature statistics - entropy (ENT), inverse difference moment (IDM), contrast (CON), correlation (COR), and angular second moment (ASM) — to represent texture [31].

The specific calculation process of texture features is as follows: First, the RGB image is converted to color space, and its corresponding value in the HSD and HSP space models is calculated. In this study, the H, S, D, and P components were non-uniformly quantized into eight parts in order to reduce information redundancy and make the calculation of the co-occurrence matrix $(\mathrm{CCM})$ easier. When calculating the CCM, set the four components as $C_{1}, C_{2}, C_{3}$, and $C_{4}$, set $m=C_{k}, n=C_{k^{\prime}}$ as the two components in the four-component combination space $\left(k, k^{\prime} \in\{1,2,3\}\right)$, and use the color co-occurrence matrix $C C M_{m n}$ to represent the different components, $C_{k}$ and $C_{k^{\prime}}$, of the pixels in the image; specifically, the measurement of the interaction between $m$ and $n$ space. From this, $C C M_{H H}$, $C C M_{S S}, C C M_{P P}, C C M_{D D}, C C M_{H S}, C C M_{H P}, C C M_{S P}, C C M_{H D}$, and $C C M_{S D}$ can be calculated. Since the symbiosis matrices $C C M_{H H}, C C M_{S S}, C C M_{P P}$, and $C C M_{D D}$ only have elements on the diagonal, these elements have little effect on distinguishing various textures, and thus the remaining four matrices are used for calculation. For any pixel in the image, assuming that the value of its $k$ th component is $i$, i.e., $m=i$, and the value of the $k^{\prime}$ th component is $j$, i.e., $n=j$, then the element $C C M_{m . n}(i, j)$ in the matrix is used to represent the number of occurrences of such pixels in the image. The calculation formula of the color co-occurrence matrix is as follows:

$$
\operatorname{CCM}_{m, n}(i, j)=\sum_{\Delta x} \sum_{\Delta y} \begin{cases}1 & m(x+\Delta x, y+\Delta y)=i \& n(x+\Delta x, y+\Delta y)=j) \\ 0 & \text { others }\end{cases}
$$

where the $i$ th row and $j$ th column elements in the color co-occurrence matrix are represented by $p(i, j)$.

\subsection{Evaluation Index of the Fused Image}

Two objective indicators-entropy and cross-entropy-were used to evaluate the quality of the fused images. Before image evaluation, each image in the HSI space is converted to an RGB image, and the grayscale operation is performed. The entropy of an image (E) is an important index for measuring the richness of image information. The larger the entropy, the more information the image has. Cross-entropy $(\mathrm{C})$ can be used to determine the information difference between the grayscale distribution of two images and is a key index for evaluating the difference between two images. 
The smaller the cross-entropy, the smaller the difference between the fused image and the source image, and the more information the fusion method extracts from the source image. The calculation formulas of entropy and cross-entropy, respectively, are:

$$
\begin{aligned}
& E=\sum_{i=0}^{L-1} p_{i} \log _{2} p_{i}, \\
& C=\sum_{i=0}^{L-1} p_{i} \log _{2} \frac{p_{i}}{q_{i}},
\end{aligned}
$$

where $L$ represents the grayscale level of the image, which is 256 , and $p_{i}$ and $q_{i}$ represent the ratio of pixels with grayscale value to the total pixels in the source image and fused image, respectively.

\subsection{Model Establishment and Evaluation Methods}

\subsubsection{Evaluation Method of the Hole Repair Algorithm}

In order to evaluate the effectiveness and accuracy of the hole repair algorithm, we artificially created holes in existing depth images, including local holes and large-area holes at the edges, and evaluated the algorithm by calculating the true value and the RMSE of the filling value calculated by the algorithm. The calculation formula of the RMSE is as follows, in which $M_{i}$ represents the repair value of each hole point, $T_{i}$ represents the true value corresponding to the hole point, and $n$ represents the number of hole points:

$$
\text { RMSE }_{\text {hole }}=\sqrt{\frac{1}{n}\left(\sum_{i=1}^{n}\left(M_{i}-T_{i}\right)^{2}\right)} .
$$

\subsubsection{Establishment and Evaluation Method of the Wheat Nitrogen Accumulation Model}

Due to high feature dimensionality, we selected two methods-multiple regression analysis and the BP neural network-to separately construct the model. In order to reduce the phenomenon of overfitting and improve the generalization ability of the model, the prediction result of the BP neural network model was the mean value of five trials, in which $50 \%$ of the samples were randomly selected each time as a training set (14 for a single growth period, and 54 for a full growth period), $20 \%$ as the validation set (five for a single growth period and 22 for a full growth period), and $30 \%$ as a test set (eight for a single growth period and 32 for a full growth period). The multiple linear regression model is as follows:

$$
y=\sum_{i=1}^{n u m} a_{i} x_{i}+b,
$$

where num is the number of different features, $x_{i}$ represents the values of different features, $y$ represents the values of LNA and SNA, and $a_{i}$ and $b$ represent the parameters of the model. The structure of the BP neural network is presented in Figure 4. There are 10 neurons in the hidden layer, and the training function is Bayesian regularization. The model was evaluated by calculating $\mathrm{R}^{2}$ and RRMSE. The calculation formulas of the RMSE and RRMSE are as follows:

$$
\begin{gathered}
\text { RMSE }=\sqrt{\frac{1}{N} \sum_{i=1}^{N}\left(y_{p i}-y_{r i}\right)^{2}}, \\
\operatorname{RRMSE}(\%)=\frac{100}{\overline{y_{r}}} \sqrt{\frac{1}{N} \sum_{i=1}^{N}\left(y_{p i}-y_{r i}\right)^{2}},
\end{gathered}
$$

where $y_{p i}, y_{r i}$, and $\overline{y_{r}}$ represent the predicted value, measured value, and mean value of the wheat LNA and SNA, respectively, and $N$ is the sample size. 


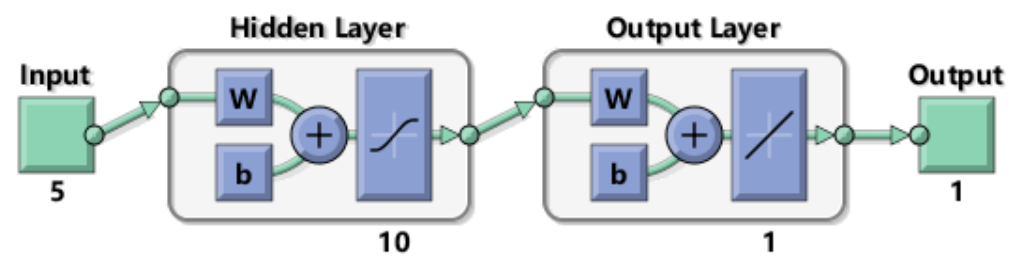

Figure 4. BP neural network structure diagram.

\section{Results and Analysis}

\subsection{Hole-Repair Results}

Since the $\mathrm{T}$ value setting in the HF algorithm will directly affect the classification results of the holes, it will thus affect the accuracy of the subsequent hole repair. In order to determine the best difference threshold $\mathrm{T}$ for the hole-repair algorithm, we employed different $\mathrm{T}$ values for experimentation. The results are listed in Table 1 . We chose a $T$ value of 40 to repair the entire depth image. Figure $5 \mathrm{~b}$ shows the repair result of the hole information. Compared with the original image shown in Figure $5 \mathrm{a}$, it is obvious that the repaired image has no black holes, and the repair quality is good. By comparing Figure $5 c, d$, i.e., before and after the repair, it can clearly be seen that the edge information (the transition of depth information) and the information of the local hole have been repaired, and the overall curve is smooth.

Table 1. Hole-repair algorithm results.

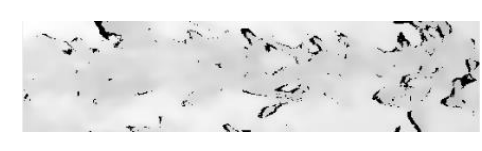

(a)

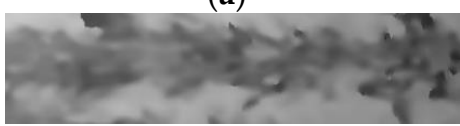

(b)

\begin{tabular}{cc}
\hline $\mathbf{T}(\mathbf{m m})$ & RMSE $_{\text {hole }}$ \\
\hline 20 & 21.09 \\
30 & 20.32 \\
40 & 11.44 \\
50 & 14.59 \\
\hline
\end{tabular}

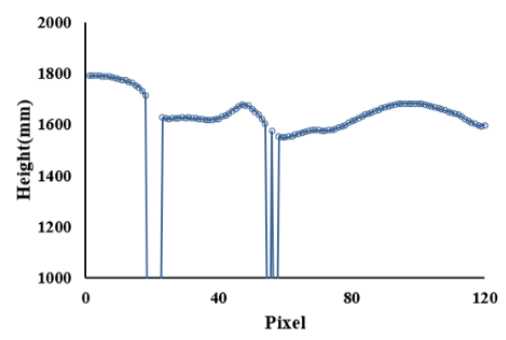

(c)

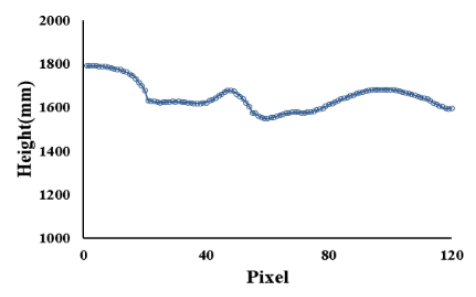

(d)

Figure 5. Depth image hole-repair results: (a) grayscale depth image; (b) repaired depth image; $(\mathbf{c}, \mathbf{d})$ original depth information and repair result of the 16th column of the image, respectively. (the grayscale difference between $(\mathbf{a}, \mathbf{b})$ is due to the fact that the grayscale range is inconsistent when the image is displayed.).

\subsection{Results of Wheat Canopy Segmentation}

The basic idea of the traditional color vegetation index for canopy segmentation is to use the difference between color information of the crop row and soil background, although the segmentation effect will be affected by factors such as light conditions and weeds. We converted the RGB color model to an HSI color model and performed canopy segmentation using the definition of hue to reduce the impact of light changes on crop row extraction. In addition, the depth information was used 
to remove weeds, thus reducing the interference of the green background of the non-wheat canopy on the extraction of crop rows. By analyzing the heights of wheat and weeds at the jointing stage, the maximum height of wheat and the average height of weeds under different nitrogen treatments were counted. The results are shown in Figure 6. Taking into account the height difference of the leaves at different positions of the wheat, only the pixels below $10 \%$ of the maximum height were regarded as weed information and removed. Figure 7 shows the crop row information extracted by the ExG and ExGR vegetation indices. It can be seen that the reflection of the leaves on the right side of the image has a poor segmentation effect. Figure $7 \mathrm{~b}$ shows the canopy segmentation resulting from simultaneously removing the soil background and weed information, which is better than the segmentation effect of the vegetation index that only depends on color features. As seen in the figure, the outline of the crop rows is clear, and the reflective part of the leaves does not affect the segmentation.

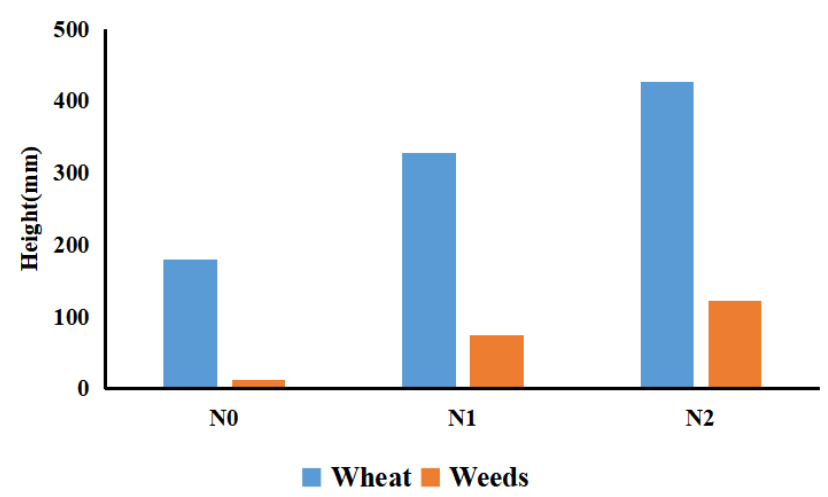

Figure 6. Height information of wheat and weeds under different treatments.

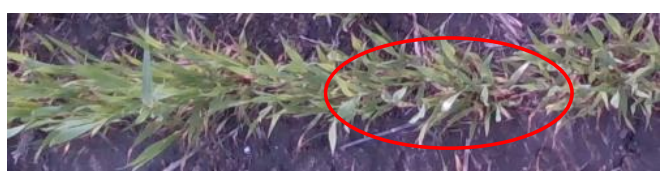

(a)

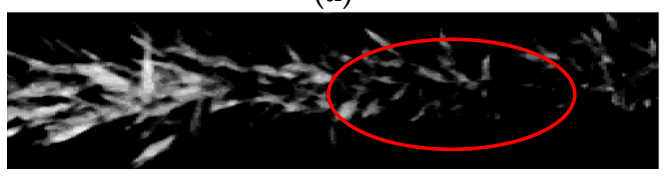

(c)

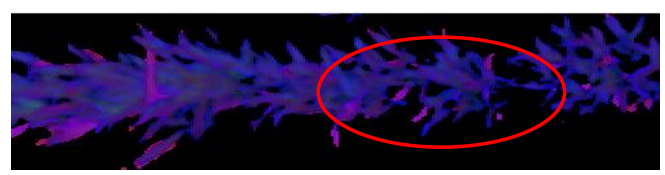

(b)

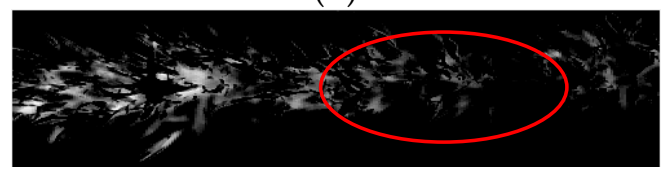

(d)

Figure 7. Soil background segmentation results: (a) RGB image; (b) crop row information extracted based on the HSI model; (c,d) crop row information extracted by the ExG and ExGR vegetation indices, respectively. The red oval indicates the reflective part of the leaves in the crop row.

\subsection{Fused Image Quality Analysis}

Figure 8 shows the image fusion results under low light, strong light, and normal light. Table 2 shows the quality evaluation results of fused images under different lighting conditions, in which fused image 1 represents the fused image based on phase information, and fused image 2 represents the fused image based on depth information. The calculation results reveal that the entropy of the fused image under different lighting conditions is greater than that of the original image, while the cross-entropy is smaller, indicating that the quality of the fused image has been improved and the information of the original image has been better retained. 


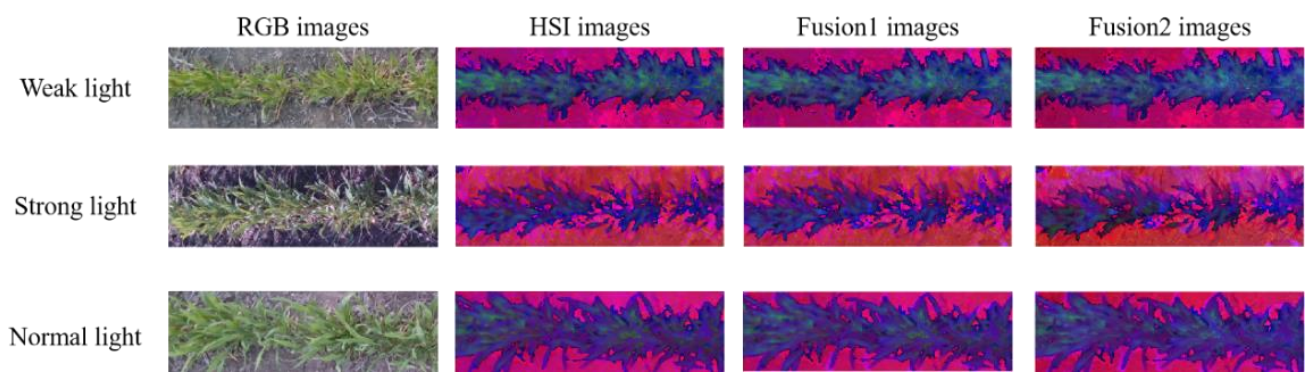

Figure 8. Image fusion results under different lighting conditions.

Table 2. Entropy and cross-entropy of source image and fused images.

\begin{tabular}{ccccc}
\hline & Quality & Weak Light & Strong Light & Normal Light \\
\hline Original image & Entropy & 6.6657 & 6.8190 & 6.7336 \\
Fusion image1 & Entropy & 6.9854 & 7.1891 & 7.1824 \\
& Cross-entropy & 0.1318 & 0.0896 & 0.0854 \\
Fusion image2 & Entropy & 6.7133 & 6.8487 & 6.8019 \\
& Cross-entropy & 0.1518 & 0.1147 & 0.1827 \\
\hline
\end{tabular}

\subsection{Forecast Results of Wheat Nitrogen Accumulation}

\subsubsection{Feature Screening of Fused Images of the Wheat Canopy}

A total of 25 features were extracted from the color co-occurrence matrix of two fused images. The feature data was linearly fitted with the wheat LNA and SNA, and feature screening was performed by comparing the $\mathrm{R}^{2}$ magnitude and the results are shown in Figure 9. In order to select textural features that have strong applicability in different growth periods, four features of angular second moment and entropy derived from the $\mathrm{CCM}_{\mathrm{HP}}$ and $\mathrm{CCM}_{\mathrm{HD}}$ were selected in the early jointing, late jointing, and booting stages. During the heading stage, however, the canopy structure changes significantly due to the wheat heading. Four features were selected, including the contrast derived from the $\mathrm{CCM}_{\mathrm{HS}}$, the second moment and entropy of the $\mathrm{CCM}_{\mathrm{HP}}$ symbiosis matrix angle, and the entropy calculated in the $\mathrm{CCM}_{\mathrm{HD}}$.

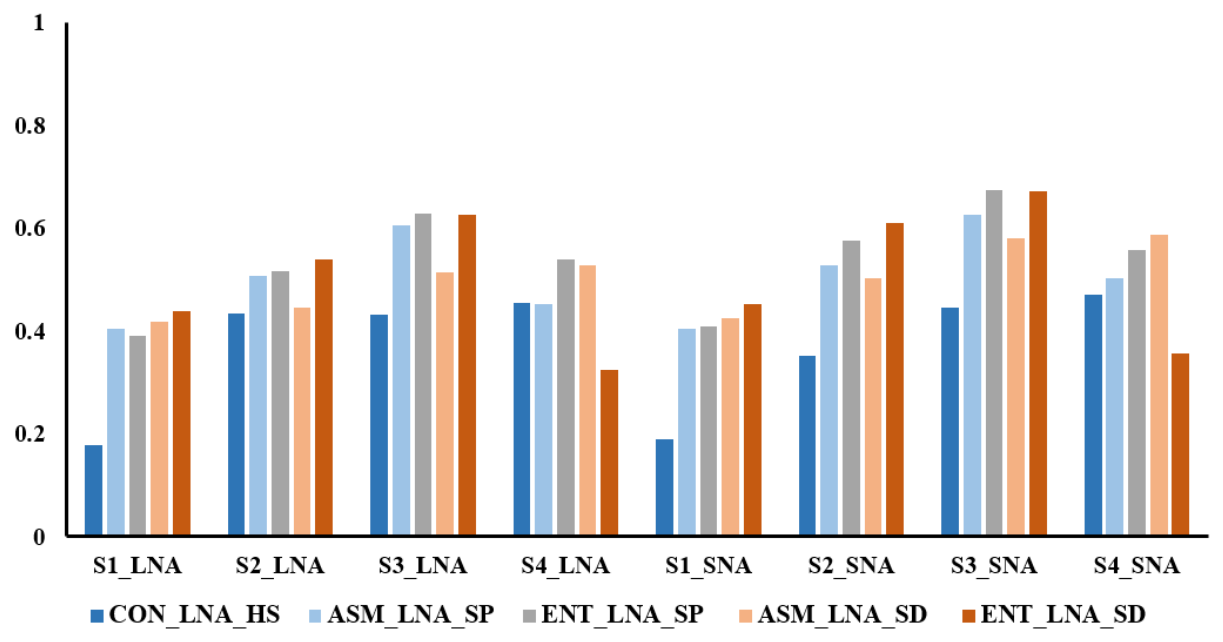

Figure 9. Feature screening results, in which S1, S2, S3, and S4 represent the early jointing, late jointing, booting, and heading stages of wheat, respectively.

\subsubsection{Forecast Results of Nitrogen Accumulation in Wheat during Different Growth Periods}

Table 3 shows the prediction ability of wheat LNA and SNA with different features during the early stage of jointing. By comparing the prediction results of different features, it was discerned that 
the prediction accuracy based on spectral features is significantly lower than that based on fusion features. This is because in the early stage of jointing, wheat plants are small, and soil background has a huge impact on the collection of canopy reflectivity, resulting in low prediction accuracy. Since the features extracted by image fusion are not affected by soil background, the prediction effect of fused image features is better than that of the spectral features.

Table 3. Comparison of LNA and SNA estimates derived from different features in the early jointing stage, in which F1 represents the feature set extracted based on the fused image, F2 represents the set based on the fused image features and the RVI, and F3 represents the set based on the fused image features and the NDRE.

\begin{tabular}{cccccccccc}
\hline \multicolumn{1}{c}{ LNA } & \multicolumn{1}{c}{ SNA } \\
\hline Feature & Model & $\mathbf{R}^{\mathbf{2}}$ & RMSE & RRMSE & Feature & Model & R $^{\mathbf{2}}$ & RMSE & RRMSE \\
\hline NDRE & LR & 0.37 & 1.03 & $45.47 \%$ & NDRE & LR & 0.31 & 2.92 & $47.91 \%$ \\
RVI & LR & 0.30 & 1.09 & $48.12 \%$ & RVI & LR & 0.24 & 3.04 & $49.88 \%$ \\
F1 & MR & 0.63 & 0.85 & $37.53 \%$ & F1 & MR & 0.63 & 2.28 & $37.41 \%$ \\
F1 & BP & 0.69 & 0.93 & $41.06 \%$ & F1 & BP & 0.67 & 2.33 & $38.23 \%$ \\
F2 & MR & 0.65 & 0.84 & $37.08 \%$ & F2 & MR & 0.63 & 2.31 & $37.90 \%$ \\
F2 & BP & 0.72 & 0.94 & $41.50 \%$ & F2 & BP & 0.70 & 2.24 & $36.75 \%$ \\
F3 & MR & 0.66 & 0.83 & $36.64 \%$ & F3 & MR & 0.64 & 2.29 & $37.57 \%$ \\
F3 & BP & 0.70 & 0.89 & $39.29 \%$ & F3 & BP & 0.69 & 2.36 & $38.72 \%$ \\
\hline
\end{tabular}

As the wheat grows and forms joints, it gradually closes the rows, and the collection of canopy reflectance is no longer disturbed by soil background. Tables 4-6 present the prediction results of different characteristics in the late jointing, booting, and heading stages, respectively. The prediction accuracy of wheat LNA and SNA based on spectral characteristics during these stages is higher and tends to be stable. Meanwhile, the prediction accuracy based on fused image features is close to the prediction accuracy during the early stage of jointing, indicating that the canopy structure features reflected by fused images are not affected by the growth period and are relatively consistent in predicting wheat LNA and SNA at different growth stages.

By comparing the prediction results of wheat LNA and SNA based on fused image features at different growth stages listed in Tables 3-6 it was found that the prediction of SNA based on fused image features is better. This is because the depth information in the fused image not only contains the structure of the wheat leaf layer, but also reflects the height of the wheat stalk, so it can more accurately express the nitrogen accumulation of the aboveground wheat population.

Table 4. Comparison of LNA and SNA estimates derived from different features in the late jointing stage.

\begin{tabular}{cccccccccc}
\hline & \multicolumn{3}{c}{ LNA } & \multicolumn{5}{c}{ SNA } \\
\hline Feature & Model & $\mathbf{R}^{\mathbf{2}}$ & RMSE & RRMSE & Feature & Model & R $^{\mathbf{2}}$ & RMSE & RRMSE \\
\hline NDRE & LR & 0.68 & 0.97 & $30.30 \%$ & NDRE & LR & 0.72 & 1.24 & $24.20 \%$ \\
RVI & LR & 0.70 & 0.93 & $29.05 \%$ & RVI & LR & 0.72 & 1.23 & $24.01 \%$ \\
F1 & MR & 0.58 & 1.17 & $36.54 \%$ & F1 & MR & 0.62 & 1.63 & $31.81 \%$ \\
F1 & BP & 0.69 & 1.07 & $33.42 \%$ & F1 & BP & 0.71 & 1.57 & $30.64 \%$ \\
F2 & MR & 0.76 & 0.91 & $28.42 \%$ & F2 & MR & 0.80 & 1.20 & $23.42 \%$ \\
F2 & BP & 0.86 & 1.00 & $31.23 \%$ & F2 & BP & 0.88 & 1.09 & $21.27 \%$ \\
F3 & MR & 0.75 & 0.93 & $29.05 \%$ & F3 & MR & 0.81 & 1.19 & $23.23 \%$ \\
F3 & BP & 0.81 & 0.92 & $28.73 \%$ & F3 & BP & 0.86 & 1.06 & $20.69 \%$ \\
\hline
\end{tabular}


Table 5. Comparison of LNA and SNA estimates derived from different features in the booting stage.

\begin{tabular}{cccccccccc}
\hline \multicolumn{1}{c}{ LNA } & \multicolumn{1}{c}{ SNA } \\
\hline Feature & Model & $\mathbf{R}^{\mathbf{2}}$ & RMSE & RRMSE & Feature & Model & R $^{\mathbf{2}}$ & RMSE & RRMSE \\
\hline NDRE & LR & 0.69 & 2.00 & $35.19 \%$ & NDRE & LR & 0.71 & 2.90 & $31.57 \%$ \\
RVI & LR & 0.70 & 1.99 & $35.01 \%$ & RVI & LR & 0.74 & 2.90 & $31.57 \%$ \\
F1 & MR & 0.71 & 2.08 & $36.60 \%$ & F1 & MR & 0.71 & 3.09 & $33.64 \%$ \\
F1 & BP & 0.76 & 1.70 & $29.91 \%$ & F1 & BP & 0.82 & 2.16 & $23.52 \%$ \\
F2 & MR & 0.76 & 1.93 & $33.96 \%$ & F2 & MR & 0.78 & 2.78 & $30.27 \%$ \\
F2 & BP & 0.81 & 1.35 & $23.75 \%$ & F2 & BP & 0.89 & 1.95 & $21.23 \%$ \\
F3 & MR & 0.76 & 1.92 & $33.78 \%$ & F3 & MR & 0.78 & 2.76 & $30.05 \%$ \\
F3 & BP & 0.80 & 1.99 & $35.01 \%$ & F3 & BP & 0.83 & 2.38 & $25.91 \%$ \\
\hline
\end{tabular}

Table 6. Comparison of LNA and SNA estimates derived from different features in the heading stage.

\begin{tabular}{cccccccccc}
\hline & \multicolumn{3}{c}{ LNA } & \multicolumn{5}{c}{ SNA } \\
\hline Feature & Model & $\mathbf{R}^{\mathbf{2}}$ & RMSE & RRMSE & Feature & Model & R $^{\mathbf{2}}$ & RMSE & RRMSE \\
\hline NDRE & LR & 0.71 & 1.68 & $38.19 \%$ & NDRE & LR & 0.77 & 2.87 & $32.08 \%$ \\
RVI & LR & 0.74 & 1.61 & $36.60 \%$ & RVI & LR & 0.79 & 2.71 & $30.29 \%$ \\
F1 & MR & 0.63 & 2.04 & $46.37 \%$ & F1 & MR & 0.79 & 2.62 & $29.28 \%$ \\
F1 & BP & 0.74 & 1.60 & $36.37 \%$ & F1 & BP & 0.78 & 2.49 & $27.83 \%$ \\
F2 & MR & 0.76 & 1.93 & $43.87 \%$ & F2 & MR & 0.84 & 2.48 & $27.72 \%$ \\
F2 & BP & 0.87 & 1.17 & $26.59 \%$ & F2 & BP & 0.88 & 2.36 & $26.38 \%$ \\
F3 & MR & 0.77 & 1.65 & $37.51 \%$ & F3 & MR & 0.82 & 2.79 & $31.18 \%$ \\
F3 & BP & 0.84 & 1.40 & $31.82 \%$ & F3 & BP & 0.87 & 2.18 & $24.36 \%$ \\
\hline
\end{tabular}

Tables 3-7 show the prediction accuracy of different features in different models. In all growth periods, the prediction results of the BP neural network were found to be better than those of the multiple regression model. There is an incomplete linear correlation between the fused image features and LNA and SNA, and since the multiple regression model cannot accurately describe the nonlinear correlation, the result is a certain deviation. The prediction results of all growth periods reveal that the combination of RVI and fused image features performs best, with $\mathrm{R}^{2}>0.7$ in the BP neural network. In addition, in the multiple regression model, the integration of the fused image features also significantly improved the prediction accuracy of the spectral features for LNA and SNA. This proves that the fused image features expressing canopy structure parameters can compensate for the LNA and SNA prediction models based on spectral features to a certain extent, and effectively improve the prediction accuracy of the models.

Table 7. Comparison of LNA and SNA estimates derived from different features in all stages.

\begin{tabular}{cccccccccc}
\hline \multicolumn{1}{c}{ LNA } & \multicolumn{5}{c}{ SNA } \\
\hline Feature & Model & $\mathbf{R}^{\mathbf{2}}$ & RMSE & RRMSE & Feature & Model & R $^{\mathbf{2}}$ & RMSE & RRMSE \\
\hline NDRE & LR & 0.63 & 1.75 & $45.02 \%$ & NDRE & LR & 0.56 & 3.16 & $43.42 \%$ \\
RVI & LR & 0.66 & 1.68 & $43.22 \%$ & RVI & LR & 0.59 & 3.05 & $41.91 \%$ \\
F1 & MR & 0.46 & 2.12 & $54.53 \%$ & F1 & MR & 0.54 & 3.29 & $45.21 \%$ \\
F1 & BP & 0.61 & 2.15 & $55.31 \%$ & F1 & BP & 0.66 & 2.83 & $38.89 \%$ \\
F2 & MR & 0.72 & 1.53 & $39.36 \%$ & F2 & MR & 0.70 & 2.66 & $36.55 \%$ \\
F2 & BP & 0.74 & 1.56 & $40.13 \%$ & F2 & BP & 0.73 & 2.60 & $35.73 \%$ \\
F3 & MR & 0.70 & 1.58 & $40.64 \%$ & F3 & MR & 0.68 & 2.72 & $37.38 \%$ \\
F3 & BP & 0.77 & 1.29 & $33.18 \%$ & F3 & BP & 0.71 & 2.67 & $36.69 \%$ \\
\hline
\end{tabular}




\section{Discussion}

\subsection{Prediction Model Performance of Wheat Nitrogen Accumulation Based on Spectral Characteristics}

In previous studies, multispectral remote sensing technology has been used to estimate the amount of nitrogen accumulation in wheat [32,33]. It was found that in the range $\mathrm{f} 50-750 \mathrm{~nm}$ for winter wheat, the spectral reflectance of the canopy changes with the change of leaf nitrogen content [34]. The spectral information in the red light portion of the spectrum is often used as an index for predicting nitrogen content [35], which proves the potential of spectral features to predict nitrogen accumulation in wheat. However, the spectral reflectance of the crop canopy exhibits obvious anisotropic characteristics due to the differences in canopy structure. The nitrogen accumulation prediction model based on spectral features ignores the influence of canopy structure on spectral information, resulting in low prediction accuracy and insufficient stability of prediction models $[7,13,34]$. In our study, depth images and RGB images were combined to extract wheat canopy texture features with depth information, and wheat canopy structure information was expressed from a three-dimensional perspective. The experimental results revealed that when predicting the nitrogen accumulation in wheat, the three-dimensional structural characteristics of the wheat canopy compensated for the shortcomings of the nitrogen accumulation prediction model, effectively improving the prediction accuracy of the model.

\subsection{Expression Method of Wheat Canopy Structure Based on RGB-D Fused Images}

Image fusion is generally divided into three levels: pixel-level, feature-level, and decision-level. At present, in most studies, feature-level fusion methods have often been used when predicting crop canopy structure parameters with RGB-D data [36,37], i.e., extracting features from RGB images and depth images separately, and predicting parameters by combining features. This method, however, often ignores the relationship between the corresponding pixels in the RGB images and depth images. The pixel-level fusion method can retain as much detailed information of the original image as possible. After fusing depth images with RGB images at the pixel level in our study, the extracted fused image features contained the depth information of crop rows, which could break through the limitations of two-dimensional images and express canopy structure information of wheat more accurately. The extracted fused image features cannot quantitatively describe canopy structure information, however. In subsequent research, we will continue to explore the extraction of more detailed canopy structure features from point cloud information, such as leaf inclination.

\subsection{Generalization Ability of the Wheat Nitrogen Accumulation Prediction Model}

The generalization ability of a model refers to its ability to adapt to fresh samples. The generalization ability directly determines the stability and versatility of the model. We utilized the independent experimental data of a wheat variety under three nitrogen treatments and two density treatments to construct and verify the model, and to improve the generalization ability of the model through cross-validation. When applying the model to other varieties, however, the model parameters still need to be corrected. This is because the plant height, leaf inclination angle, tiller number, and leaf development process of different varieties are quite different. In the follow-up study, different plant types of wheat varieties will be tested in order to further improve the generalization ability of the model among different varieties of wheat.

\section{Conclusions}

We proposed a pixel-level RGB-D image fusion method, which expresses three-dimensional structural information of the canopy by extracting textural features of fused images, thereby compensating the model for predicting nitrogen accumulation based only on spectral characteristics. We evaluated and analyzed the compensation effect by using two models-multiple regression analysis and the BP neural network. Our work is summarized as follows: 
(1) We proposed a hole-filling algorithm with depth information. When $\mathrm{T}=40 \mathrm{~mm}$, the repair accuracy is the best (RMSE $=11.44$ ), effectively avoiding the loss of features due to information loss.

(2) We used IHS transformation to fuse RGB images and depth images. The textural features of the fused images contain depth information, which can break through the limitation of extracting canopy structure features from a two-dimensional image and express the three-dimensional structure information of wheat canopy.

(3) Our test results revealed that in different models, the combination of the characteristics of the fused image with the spectral characteristics can predict nitrogen accumulation in wheat more accurately. In the prediction of a full growth period, the best prediction accuracy values $\left(\mathrm{R}^{2}\right)$ of the combined features for LNA and SNA were 0.74 and 0.73 , respectively, with corresponding RRMSEs of $40.13 \%$ and $35.73 \%$, indicating good predictive ability.

Author Contributions: J.N., Y.Z., X.J. and W.C. designed the research, J.N., K.X. and J.Z. developed the algorithms, H.L. and K.X. performed the research, K.X. and J.Z. analyzed the data and wrote the paper. All authors have read and agreed to the published version of the manuscript.

Funding: This work was supported in part by the National Key Research and Development Program of China (Grant No. 2017YFD0201501), National Natural Science Foundation of China (Grant No. 31871524), Six Talent Peaks Project in Jiangsu Province (Grant No. XYDXX-049), the Primary Research and Development Plan of Jiangsu Province of China (BE2017385, BE2018399, BE2019306), Jiangsu Graduate Innovation Program (KYCX20_0575), and the 111 Project (B16026).

Acknowledgments: The authors wish to thank all those who helped in this research.

Conflicts of Interest: The authors declare no conflict of interest.

\section{References}

1. Sutton, M.A.; Oenema, O.; Erisman, J.W.; Leip, A.; van Grinsven, H.; Winiwarter, W. Too much of a good thing. Nature 2011, 472, 159-161. [CrossRef]

2. Hatfield, J.L.; Gitelson, A.A.; Schepers, J.S.; Walthall, C.L. Application of Spectral Remote Sensing for Agronomic Decisions. Agron. J. 2015, 100, 117-131. [CrossRef]

3. Lemaire, G.; Jeuffroy, M.H.; Gastal, F. Diagnosis tool for plant and crop N status in vegetative stage: Theory and practices for crop N management. Eur. J. Agron. 2008, 28, 614-624. [CrossRef]

4. Filella, I.; Serrano, L.; Serra, J.; Peuelas, J. Evaluating Wheat Nitrogen Status with Canopy Reflectance Indices and Discriminant Analysis. Crop Sci. 1995, 35, 1400-1405. [CrossRef]

5. Hansen, P.; Schjoerring, J.K. Reflectance measurement of canopy biomass and nitrogen status in wheat crops using normalized difference vegetation indices and partial least squares regression. Remote Sens. Environ. 2003, 86, 542-553. [CrossRef]

6. Karcher, D.E.; Richardson, M.D. Quantifying Turfgrass Color Using Digital Image Analysis. Crop Sci. 2003, 43, 943-951. [CrossRef]

7. Lee, K.J.; Lee, B.-W. Estimation of rice growth and nitrogen nutrition status using color digital camera image analysis. Eur. J. Agron. 2013, 48, 57-65. [CrossRef]

8. Broge, N.H.; Mortensen, J.V. Deriving green crop area index and canopy chlorophyll density of winter wheat from spectral reflectance data. Remote Sens. Environ. 2002, 81, 45-57. [CrossRef]

9. Berger, K.; Verrelst, J.; Féret, J.-B.; Wang, Z.; Hank, T. Crop nitrogen monitoring: Recent progress and principal developments in the context of imaging spectroscopy missions. Remote Sens. Environ. 2020, 242, 111758. [CrossRef]

10. Gracia-Romero, A.; Kefauver, S.C.; Vergara-Diaz, O.; Zaman-Allah, M.A.; Prasanna, B.M.; Cairns, J.E.; Araus, J.L. Comparative Performance of Ground vs. Aerially Assessed RGB and Multispectral Indices for Early-Growth Evaluation of Maize Performance under Phosphorus Fertilization. Front. Plant Sci. 2017, 8 , 2004. [CrossRef]

11. Sankaran, S.; Khot, L.R.; Carter, A.H. Field-based crop phenotyping: Multispectral aerial imaging for evaluation of winter wheat emergence and spring stand. Comput. Electron. Agric. 2015, 118, 372-379. [CrossRef] 
12. Diacono, M.; Rubino, P.; Montemurro, F. Precision nitrogen management of wheat. A review. Agron. Sustain. Dev. 2013, 33, 219-241. [CrossRef]

13. Singh, A.; Ganapathysubramanian, B.; Singh, A.K.; Sarkar, S. Machine learning for high-throughput stress phenotyping in plants. Trends Plant Sci. 2016, 21, 110-124. [CrossRef]

14. Knyazikhin, Y.; Schull, M.A.; Stenberg, P.; Mottus, M.; Rautiainen, M.; Yang, Y.; Marshak, A.; Carmona, P.L.; Kaufmann, R.K.; Lewis, P. Hyperspectral remote sensing of foliar nitrogen content. Proc. Natl. Acad. Sci. USA 2012, 110, E185-E192. [CrossRef] [PubMed]

15. Gamon, J.A.; Field, C.B.; Goulden, M.L.; Griffin, K.L.; Hartley, A.E.; Joel, G.; Peñuelas, J.; Valentini, R. Relationships between NDVI, canopy structure, and photosynthesis in three Californian vegetation types. Ecol. Appl. 1995, 5, 28-41. [CrossRef]

16. Rorie, R.L.; Purcell, L.C.; Karcher, D.E.; King, C.A. The Assessment of Leaf Nitrogen in Corn from Digital Images. Crop Sci. 2011, 51, 2174-2180. [CrossRef]

17. Fernández, E.; Gorchs, G.; Serrano, L.; Lightfoot, D.A. Use of consumer-grade cameras to assess wheat $\mathrm{N}$ status and grain yield. PLoS ONE 2019, 14, e0211889. [CrossRef]

18. Fernandez-Gallego, J.A.; Kefauver, S.C.; Gutiérrez, N.A.; Nieto-Taladriz, M.T.; Araus, J.L. Wheat ear counting in-field conditions: High throughput and low-cost approach using RGB images. Plant Methods 2018, 14, 22. [CrossRef]

19. Wang, Y.; Wang, D.; Shi, P.; Omasa, K. Estimating rice chlorophyll content and leaf nitrogen concentration with a digital still color camera under natural light. Plant Methods 2014, 10,1-11. [CrossRef]

20. Li, L.; Zhang, Q.; Huang, D. A Review of Imaging Techniques for Plant Phenotyping. Sensors 2014, 14, 20078-20111. [CrossRef]

21. Jay, S.; Rabatel, G.; Hadoux, X.; Moura, D.; Gorretta, N. In-field crop row phenotyping from 3D modeling performed using Structure from Motion. Comput. Electron. Agric. 2015, 110, 70-77. [CrossRef]

22. Andujar, D.; Dorado, J.; Bengochea-Guevara, J.M.; Conesa-Munoz, J.; Fernandez-Quintanilla, C.; Ribeiro, A. Influence of Wind Speed on RGB-D Images in Tree Plantations. Sensors 2017, 17, 914. [CrossRef] [PubMed]

23. Jiang, Y.; Li, C.; Paterson, A.H.; Sun, S.; Xu, R.; Robertson, J. Quantitative Analysis of Cotton Canopy Size in Field Conditions Using a Consumer-Grade RGB-D Camera. Front. Plant Sci. 2018, 8, 2233. [CrossRef] [PubMed]

24. Andújar, D.; Ribeiro, A.; Fernández-Quintanilla, C.; Dorado, J. Using depth cameras to extract structural parameters to assess the growth state and yield of cauliflower crops. Comput. Electron. Agric. 2016, 122, 67-73. [CrossRef]

25. Peteinatos, G.G.; Weis, M.; Andújar, D.; Ayala, V.R.; Gerhards, R. Potential use of ground-based sensor technologies for weed detection. Pest Manag. Sci. 2014, 70, 190-199. [CrossRef]

26. Coy, A.; Rankine, D.; Taylor, M.; Nielsen, D.C.; Cohen, J. Increasing the Accuracy and Automation of Fractional Vegetation Cover Estimation from Digital Photographs. Remote Sens. 2016, 8, 474. [CrossRef]

27. Jiang, Q.; Fang, S.; Peng, Y.; Gong, Y.; Zhu, R.; Wu, X.; Ma, Y.; Duan, B.; Liu, J. UAV-Based Biomass Estimation for Rice-Combining Spectral, TIN-Based Structural and Meteorological Features. Remote Sens. 2019, 11, 890. [CrossRef]

28. Cai, Z.Y.; Shao, L. RGB-D Data Fusion in Complex Space. In Proceedings of the 2017 24th IEEE International Conference on Image Processing (ICIP), Beijing, China, 17-20 September 2017; pp. 1965-1969.

29. Li, H.; Lin, W.; Pang, F.; Jiang, X.; Ni, J. Monitoring Wheat Growth Using a Portable Three-Band Instrument for Crop Growth Monitoring and Diagnosis. Sensors 2020, 20, 2894. [CrossRef]

30. Feng, W.; Yao, X.; Zhu, Y.; Tian, Y.C.; Cao, W.X. Monitoring leaf nitrogen status with hyperspectral reflectance in wheat. Eur. J. Agron. 2008, 28, 394-404. [CrossRef]

31. Maheswari, G.U.; Ramar, K.; Manimegalai, D.; Gomathi, V. An adaptive region based color texture segmentation using fuzzified distance metric. Appl. Soft Comput. 2011, 11, 2916-2924. [CrossRef]

32. Li, H.J.; Zhang, Y.M.; Lei, Y.P.; Antoniuk, V.; Hu, C.S. Evaluating Different Non-Destructive Estimation Methods for Winter Wheat (Triticum aestivum L.) Nitrogen Status Based on Canopy Spectrum. Remote Sens. 2020, 12, 95. [CrossRef]

33. Yao, X.; Zhu, Y.; Tian, Y.C.; Feng, W.; Cao, W.X. Exploring hyperspectral bands and estimation indices for leaf nitrogen accumulation in wheat. Int. J. Appl. Earth Obs. Geoinf. 2010, 12, 89-100. [CrossRef]

34. He, L.; Zhang, H.Y.; Zhang, Y.S.; Song, X.; Guo, T.C. Estimating canopy leaf nitrogen concentration in winter wheat based on multi-angular hyperspectral remote sensing. Eur. J. Agron. 2016, 73, 170-185. [CrossRef] 
35. Guo, B.B.; Qi, S.L.; Heng, Y.R.; Duan, J.Z.; Zhang, H.Y.; Wu, Y.P.; Feng, W.; Xie, Y.X.; Zhu, Y.J. Remotely assessing leaf $\mathrm{N}$ uptake in winter wheat based on canopy hyperspectral red-edge absorption. Eur. J. Agron. 2017, 82, 113-124. [CrossRef]

36. Chaivivatrakul, S.; Tang, L.; Dailey, M.N.; Nakarmi, A.D. Automatic morphological trait characterization for corn plants via 3D holographic reconstruction. Comput. Electron. Agric. 2014, 109, 109-123. [CrossRef]

37. Jiang, Y.; Li, C.; Paterson, A.H. High throughput phenotyping of cotton plant height using depth images under field conditions. Comput. Electron. Agric. 2016, 130, 57-68. [CrossRef]

Publisher's Note: MDPI stays neutral with regard to jurisdictional claims in published maps and institutional affiliations.

(C) 2020 by the authors. Licensee MDPI, Basel, Switzerland. This article is an open access article distributed under the terms and conditions of the Creative Commons Attribution (CC BY) license (http://creativecommons.org/licenses/by/4.0/). 